\title{
APPLICATION OF CHI SQUARE TESTS TO FITTING OF IONIC CONDUCTIVITY
}

\author{
R. J. FRIAUF \\ Department of Physics, University of Kansas Lawrence, Kansas 66044, USA
}

\begin{abstract}
Résumé. - En relation avec une étude de l'anomalie de la conductivité à haute température dans les halogénures d'argent, plusieurs formes de tests de chi carré ont été appliquées pour déterminer la validité statistique des résultats. En premier lieu est décrit le processus habituel pour obtenir un lissage par moindres carrés de la conductivité en fonction de la température. Ensuite une discussion des erreurs expérimentales montre que les incertitudes sur la résistance, les dimensions de l'échantillon et la mesure de la température peuvent donner une erreur totale de $0,5 \%$ et que cette erreur doit être indépendante, avec une bonne approximation, de la température. Un test de chi carré est alors décrit pour la distribution des déviations dans un intervalle intermédiaire de température. Dans les cas favorables, les erreurs peuvent être décrites par une moyenne égale à 0 et une déviation standard de $0,39 \%$ dans $\mathrm{AgCl}$ et $0,43 \%$ dans $\mathrm{AgBr}$. Enfin, une deuxième forme de test de chi carré est décrite pour mesurer la validité de lissage de la fonction à adapter basée sur le modèle de conductivité par défauts de réseau. La valcur de chi carré croît rapidement lorsque le domairie de température est étendu vers les hautes températures où se produit l'anomalie. fournissant une limite supérieure de température nécessaire à un lissage satisfaisant. On conclut que l'utilisation du test de chi carré a joué un rôle important dans la présente étude et que des utilisations semblables devraient être valables dans le cadre d'autres études de diffusion et conductivité ionique.
\end{abstract}

\begin{abstract}
In connection with a study of the high temperature conductivity anomaly for the silver halides, several forms of chi square tests have been applied to determine the statistical validity of the results. First the usual procedure for obtaining a least squares fit of conductivity us, temperature is described. Next a discussion of experimental crrors shows that uncertainties in resistance, sample dimensions, and temperature measurements may give an accumulated error of around $0.5 \%$, and that this per cent error should be independent of temperature to a good first approximation. A chi square test is then described for the distribution of deviations in an intermediate temperature range, and it is shown that in favorable cases the errors can be described satisfactorily by a normal error distribution with a mean of zero and a standard deviation of $0.39 \%$ for $\mathrm{AgCl}$ and $0.43 \%$ for $\mathrm{AgBr}$. Finally a second form of chi square test is described to measure the goodness of fit of the fitting function based on the defect model for the conductivity. It is shown that the value of chi square increases rapidly as the upper end of the intermediate temperature region is extended into the higher temperature region where the anomaly occurs, thereby providing a rather clearly defined upper temperature limit for an acceptable fit. It is concluded that these uses of the chi square test have played an important role in the present study, and that similar uses should be valuable for analyzing the experimental results of other studies of diffusion and ionic conductivity.
\end{abstract}

1. Introduction. - Measurements of ionic conductivity have served as one of the foremost methods for studying point defects in ionic crystals. On this basis, along with evidence from other phenomena such as diffusion, dielectric relaxation and color centers, it is clearly established that the major defects in alkali halides are of the Schottky type, cation and anion vacancies, whereas in the silver halides Frenkel defects occur, cation vacancies and interstitial ions. The basic purpose of the investigations, of course. is to determine the atomic properties of the defects, in particular the thermodynamic parameters, namely, the enthalpy and the entropy, controlling the formation and mobility of each kind of defect.

If only one kind of mobile defect were present. the conductivity $\sigma$ should have a temperature dependence of the form [1].

$$
\sigma T=A \exp (-W / k T) .
$$

Then the usual Arrhenius plot of $\log (\sigma T)$ vs. $1 / T$ would be a straight line and the effective activation energy $W$ could be obtained directly from the slope. This sort of simple and straightforward approach was used in much of the early work, often with different slopes for the extrinsic and intrinsic ranges. Refinements were introduced by considering the contribution from both interstitial ions and cation vacancies in the silver halides [2], the association and precipitation of divalent impurities in the alkali halides [3] and eventually the contribution from anion vacancies 
at high temperatures in the alkali halides [4]. With more detailed measurements, however, it finally became apparent that there is really no appreciable region of any Arrhenius plot that is strictly a straight line, largely because of the gradual onset of the influence of the various processes just mentioned and therefore the activation energies obtained from such procedures can have only qualitative or semiquantitative significance at best.

Consequently in recent years considerable sophistication has been introduced into the analysis of measured results by applying a least squares fitting procedure, which will be described in more detail in the next section. Examples of this approach include the early work of Beaumont and Jacobs on $\mathrm{KCl}$ [5] and of Dawson and Barr on $\mathrm{KBr}$ [6], several attempts by Allnatt et al. on $\mathrm{NaCl}$ [7], [8], the treatment with anion doping by Chandra and Rolfe for $\mathrm{KCl}$ [9], $\mathrm{KBr}$ [10], $\mathrm{KI}$ [11], and $\mathrm{RbI}$ [12] and recent consideration by Corish and Jacobs of $\mathrm{AgCl}$ [13] and by Aboagye and Friauf of $\mathrm{AgCl}$ and $\mathrm{AgBr}$ [14]. The considerable expenditure of computer time, which is the reason that such a detailed analysis is now possible, is certainly justified by the increased refinement of the treatment and the improved reliability of the results.

Despite all of the advantages of the least squares method, the approach is not without its hazards. First, a fairly large number of parameters are involved. In the alkali halides there are at least eight - two each for formation, association and mobility of cation and anion vacancies - and in the silver halides the occurrence of two types of interstitialcy jumps increases the total to ten. Second, the highly non-linear form of the overall expression for the conductivity prevents any algebraic solution of the regression equations and makes necessary a search procedure in the multiparameter space. But even granting that the possible pitfalls for finding the least squares fit can be avoided and this seems to be the case in most of the published work, there are often some remaining discrepancies after the best fit is obtained. It may not be possible to separate mechanisms with nearly equal enthalpies by considering only pure crystal results as in $\mathrm{KBr}$ [6] and $\mathrm{RbCl}$ [15], it is especially difficult to determine parameters for minority carriers as in $\mathrm{KBr}$ [6], $\mathrm{AgCl}$ [13] and $\mathrm{NaCl}$ [16], sometimes this feature leads to outlandish values for these parameters, as is not uncommon in $\mathrm{NaCl}$ [7], [16] and often the values of certain parameters for different samples of the same material differ by considerably more than the statistical error for either sample considered by itself, as in $\mathrm{NaCl}$ [8] and $\mathrm{AgCl}$ [13]. It is particularly noteworthy that there often seems to be an anomalously high conductivity at temperatures approaching the melting point; small discrepancies in $\mathrm{NaCl}$ [8] and $\mathrm{KCl}$ [9], [17] have led to the suggestion of possible additional defects such as cation Frenkel defects in $\mathrm{NaCl}$ [7] or vacancy triplets in $\mathrm{KCl}$ [18] and the much larger high tem- perature anomaly in the silver halides has long been noted [1], [2], [19]. Figure 1 shows the striking nature of this anomaly in $\mathrm{AgCl}$ [14].

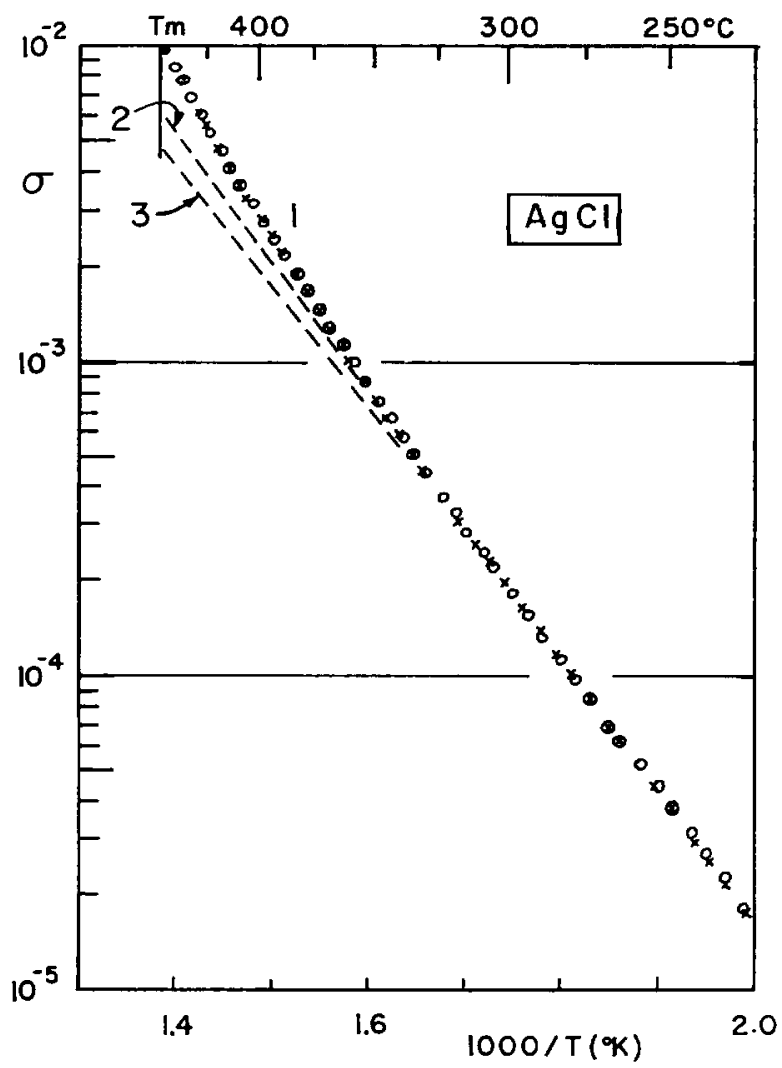

FIG. 1. - Conductivity vs. $1000 / T$ for $\mathrm{AgCl}$ [14]. 1) Experimental measurements. 2) Calculated with Debye-HückelLidiard corrections. 3) Calculated without D-H-L corrections.

The basic question in all of these cases is whether the proposed model fits the measured results within the limits of the experimental errors. A good qualitative indication is provided by the appearance of systematic, non-random trends in the deviation plots and this sort of indication is the basis for detection of most of the discrepancies mentioned above. The high temperature anomaly shows up clearly in the deviation plot for $\mathrm{AgCl}$ in figure 2, for instance, and it is clear that there is some major deficiency in the model above $300^{\circ} \mathrm{C}$. The deviation plots for $\mathrm{AgBr}$ in figure 3 are not quite so obvious, however, and in any case it would be desirable to have a more objective criterion for deciding how good a fit has been obtained. A very useful statistical measure of the "goodness of fit" is provided by the chi square test devised by Karl Pearson, and it is the purpose of this paper to describe how this test can be applied to the conductivity fitting problem.

The need for such a test arose during a detailed study of the high temperature conductivity anomaly in the silver halides [14]. See figure l. Selected data from this work will be used as examples of the application of the statistical tests discussed here. The experimental procedures, the specific values for 


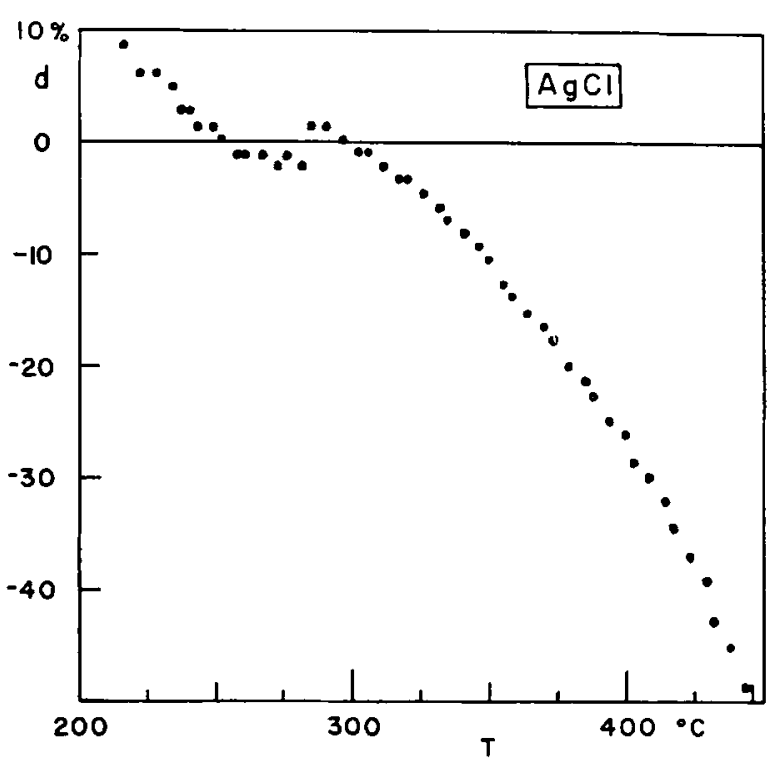

FIG. 2. - Deviation plot for $\mathrm{AgCl}$ when a fit is attempted for the complete temperature range. The deviations represent the difference between curves 2 and 1 of figure 1 .

$\mathrm{AgCl}$ and $\mathrm{AgBr}$, and the physical significance of the results are described in the other article [14].

2. Procedure for fitting conductivity. - The least squares method is applied as follows. A fairly large number of measurements of the conductivity are made at closely spaced temperatures over a wide temperature range. A model for the conductivity is established by proposing the presence of certain kinds of defects and impurities in the crystal, along with appropriate parameters for formation, mobility and association. With a trial set of parameters the conductivity is calculated at each temperature, and the deviation from the experimental value is found. The sum of the squares of the deviations is then minimized by varying the parameters until no further reduction of the sum can be obtained. The final set of parameters is declared to represent the "best fit" .

As an illustration of this procedure we summarize the appropriate equations for the silver halides. The condition of electric neutrality, the formation of intrinsic Frenkel defects, and the association equilibrium between cation vacancies and divalent impurity ions are governed by

$$
\begin{array}{cl}
x_{\mathrm{v}} & =x_{\mathrm{i}}+c-x_{\mathrm{k}}, \\
y^{2} x_{\mathrm{v}} x_{\mathrm{i}} & =2 \exp \left(-g_{\mathrm{F}} / k T\right)=x_{00}^{2}(T), \\
x_{\mathrm{k}}\left[\eta^{2} x_{\mathrm{i}}\left(c-x_{\mathrm{k}}\right)\right]^{-1} & =12 \exp (\xi / k T) \quad=K_{2}(T),
\end{array}
$$

where $x_{i}, x_{i}, c, x_{k}$ are the mole fractions of cation vacancies. interstitial silver ions. divalent impurities associated divalent impurities: and $g_{\mathrm{F}}=h_{\mathrm{F}}-T_{S_{\mathrm{F}}}$ and $\xi=\%-T \eta$ are the Gibbs free energy, enthalpy: entropy for formation and association, respectively. The long range Coulomb interactions between the charged defects are included to first order with the
Debye-Hückel-Lidiard theory [I] by defining a screening length $\kappa_{\mathrm{D}}^{-1}$, an interaction energy $\Delta g_{\mathrm{D} H}$, and an activity coefficient $\gamma$ according to

$$
\begin{aligned}
& \kappa_{\mathrm{D}}^{2}=8 \pi\left(N \mathrm{e}^{2} / k T\right)\left(x_{\mathrm{v}} / \varepsilon\right), \\
& \Delta \mathrm{g}_{\mathrm{DH}}=\mathrm{e}^{2} \kappa_{\mathrm{D}}\left[\varepsilon\left(1+\kappa_{\mathrm{D}} R\right)\right]^{-1}, \\
& \ln \gamma=-\Delta g_{\mathrm{DH}} / 2 k T,
\end{aligned}
$$

w ere $N$ is the number of silver lattice sites per unit volume, $\varepsilon$ is the dielectric constant and $R$ is the distance of closest approach in the Debye-Hückel theory. By introducing

$$
\zeta=x_{v} / x_{000}, \quad H(T)=x_{00} K_{2},
$$

and combining eq. (1), (2) and (3) we obtain

$$
\left(c / x_{00}\right)=\left(\zeta-\gamma^{-2} \zeta^{-1}\right)\left(1+\gamma^{2} \zeta H\right) \text {. }
$$

Thus by starting with $c, x_{00}$, and $K_{2}$ at some temperature, we can iterate between eq. (4) and (6) to find $\zeta$ and can then obtain $x_{v}$ and $x_{\mathrm{j}}$ from eq. (5) and (2).

The cation vacancy mobility is given by

$$
\mu_{v}=z_{v}\left(e v_{0}, a_{0}^{2} / k T\right) \exp \left(-\Delta g_{v} / k T\right),
$$

where $z_{v}=4$ is a symmetry number, $v_{0}$ is a standard lattice vibration frequency, $a_{0}$ is the nearest neighbor distance, and $\Delta g_{v}=\Delta h_{v}-T \Delta s_{v}$ are the Gibbs free energy, enthalpy, entropy associated with a vacancy jump. Similar expressions apply for the mobilities $\mu_{\mathrm{k}}$ of the collinear interstitialcy jump $\left(z_{1}=2\right)$ and $\mu_{2}$ of the non-collinear interstitialcy jump $\left(z_{2}=4\right)$. The total interstitial mobility is

$$
\mu_{\mathrm{i}}=\mu_{\mathrm{i}}+\mu_{2},
$$

and the conductivity is

$$
\sigma=\mathrm{e} N G\left(x_{\mathrm{w}} \mu_{\mathrm{w}}+x_{\mathrm{i}} \mu_{\mathrm{i}}\right),
$$

where $G$ is the Onsager-Pitts mobility drag factor $G=1-\mathrm{e}^{2} \kappa_{\mathrm{D}}\left[3 \approx k T(\sqrt{2}+1)\left(\sqrt{2}+\kappa_{\mathrm{D}} R\right)\left(1+\kappa_{\mathrm{D}} R\right)\right]^{-1}$.

In order to include results from doped conductivity and diffusion experiments, it is convenient to define the ratio $\varphi$ of interstitial to vacancy mobilities and the ratio $\kappa$ of collinear and non-collinear jump frequencies by

$$
\varphi=\mu_{i} / \mu_{v}, \quad k=2 \mu_{1 / 3} / 3 \mu_{2} .
$$

Then we find

$$
\varphi=(1+3 \kappa / 2) \varphi_{2}, \quad \mu_{i}=(1+3 \kappa / 2) \mu_{2},
$$

where $\varphi_{2}=\mu_{2} \mu_{n}$, and can write the conductionty as

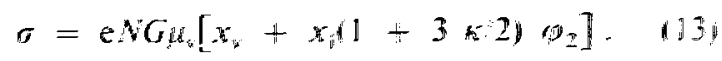

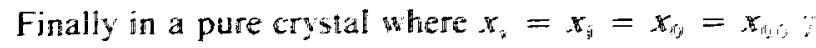
we have

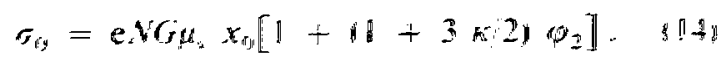


We can now summarize the least squares fitting procedure in schematic form. We start with a set of experimental measurements of $\sigma_{i}$ at corresponding temperatures $T_{\mathrm{i}}$, each measurement having, at least in principle, a known experimental error $e_{i}$. In order to take advantage of the more nearly linear form of the Arrhenius plot, we define associated variables $\left({ }^{1}\right)$

$$
y_{\mathrm{i}}=\ln \left(\sigma_{\mathrm{i}} T_{\mathrm{i}}\right), \quad x_{\mathrm{i}}=1000 / T_{\mathrm{i}} .
$$

The rather formidable looking set of eq. (1) to (10) simply says that we can calculate $\sigma$ at any $T$ when the values of all parameters are specified; this result gives us our fitting function based on the proposed model for the conductivity.

$\sigma=\sigma(T ;$

$$
\left.h_{\mathrm{F}}, s_{\mathrm{F}}, c, \chi, n, \Delta h_{\mathrm{v}}, \Delta s_{\mathrm{v}}, \Delta h_{1}, \Delta s_{1}, \Delta h_{2}, \Delta s_{2}\right) \text {. }
$$

We suppose that $a_{0}, R, N, v_{0}, \varepsilon$ are prescribed constants or functions of the temperature, but even then there are eleven (count them !) adjustable parameters. (For nearly pure crystals a better fit can be obtained by varying $c$ over a small range, a few ppm, rather than fixing $c=0$.) In terms of the associated variables we have

$$
y=f\left(x ; \lambda_{1}, \lambda_{2}, \ldots, \lambda_{r}\right),
$$

where the number of parameters $\lambda_{\mathrm{j}}$ is $r=11$ in this example. For a given set of parameters, then, we can calculate

$$
f_{\mathrm{i}}=f\left(x_{\mathrm{i}}\right), \quad d_{\mathrm{i}}=y_{\mathrm{i}}-f_{\mathrm{i}}
$$

at each experimental point.

The minimization can be applied to any function of the sum of the squares of the deviations. A commonly used expression is the best estimate of the standard deviation,

$$
(s . d .)^{2}=(n-1)^{-1} \Sigma_{i} d_{\mathrm{i}}^{2}, \quad i=1 \text { to } n,
$$

where $n$ is the number of data points. For later purposes, however, it is more appropriate to consider the variance of the fit (also see Section 5),

$$
s^{2}=(n-r)^{-1} \Sigma_{i} d_{\mathrm{i}}^{2} .
$$

We see that the number of degrees of freedom $v$ is obtained by reducing the number of data points by the number of independently adjustable parameters.

$$
v=n-r .
$$

The procedure is carried out by a trial and error variation of the parameters in order to obtain a minimum value of $s^{2}$.

(1) Although $y_{i}=\log \left(\sigma_{\mathrm{i}} T_{\mathrm{i}}\right)$ is usually used for the Arrhenius plot on semi-log graph paper, we prefer to use the natural logarithm here since then the deviations come out directly in per cent (see eq. (23))
3. Consideration of errors. - Now that we have obtained the best fit of our fitting function to the experimental observations, we should like to know how good a fit we have, i. e., what is the goodness of fit ? Before we can proceed to a direct application of the chi square test for this purpose, we must consider the nature of the information about the experimental errors. We shall see in section 5 , in fact, that in order to develop a quantitative chi square test we need to have an independent evaluation of the error $e_{i}$ in each of the data points. Hence we first consider some aspects of the errors in this section, and then show in section 4 how to use another form of chi square test to provide a quantitative measure of the errors.

The first feature of the errors arises form the logarithmic scale of the ordinate of the Arrhenius plot. If we denote the calculated value of the conductivity at $T_{\mathrm{i}}$ by $\sigma\left(T_{\mathrm{i}}\right)$, we have

$$
\begin{aligned}
d_{\mathrm{i}} & =y_{\mathrm{i}}-f_{\mathrm{i}}=\ln \left[\sigma_{\mathrm{i}} T_{\mathrm{i}}\right]-\ln \left[\sigma\left(T_{\mathrm{i}}\right) T_{\mathrm{i}}\right] \\
& =\ln \left[1+\frac{\sigma_{\mathrm{i}}-\sigma\left(T_{\mathrm{i}}\right)}{\sigma\left(T_{\mathrm{i}}\right)}\right] .
\end{aligned}
$$

In terms of the fractional error $F_{\mathrm{i}}$ (which can readily be expressed as a per cent error simply by multiplying by 100 ),

$$
F_{\mathrm{i}}=\left[\sigma_{\mathrm{i}}-\sigma\left(T_{\mathrm{i}}\right)\right] / \sigma\left(T_{\mathrm{i}}\right)
$$

we have

$$
d_{\mathrm{i}}=\ln \left(1+F_{\mathrm{i}}\right) \approx F_{\mathrm{i}},
$$

where the approximation is valid as long as $F_{\mathrm{i}} \ll 1$ (errors of a few per cent). Thus the errors $e_{i}$ that we are concerned with in the fitting program for the variables $y_{\mathrm{i}}$ are essentially fractional or per cent errors.

Next we must consider the nature of the errors entering into a conductivity experiment [8], [20]. 1) The resistance measurements are influenced by the following sources of errors. The standard decade resistors have a guaranteed accuracy of better than $0.1 \%$, the sensitivity of the bridge balance introduces an error of only 0.01 to $0.1 \%$, and the small frequency dependence of the resistance measurements is always less than $0.1 \%$. Thus the accumulated error in the resistance measurements is between 0.1 and $0.2 \%$. For most conductivity experiments, in fact, the actual measurement of the resistance is the least important source of error, provided polarization effects are avoided by using a reasonably high frequency. 2) The measurement of sample dimensions is uncertain to 0.1 to $0.2 \%$ for each dimension. giving an overall error in the geometrical factor for converting resistance to conductivity of 0.3 to $0.6 \%$ This error would remain constant for a series of measurements on one sample, but it does enter into 
consideration when data from different samples are pooled, as was sometimes done in our work. 3) There may be both systematic and random errors in the temperature measurements. Repeated calibration tests of the chromel-alumel thermocouple used for the silver halide measurements gave an internal consistency of 0.1 to $0.3^{\circ} \mathrm{C}$, and the minimum error of one count in the last digit of the digital voltmeter reading was 1 to $2 \mu \mathrm{V}$ (after subtracting the zero reading) or about $0.05{ }^{\circ} \mathrm{C}$. Systematic errors might be as large as 0.5 to $1.0^{\circ} \mathrm{C}$, but these would affect the fitting only in a more subtle way. The effect on $\sigma$ of a small error $\delta T$ can be estimated by differentiating $\sigma \approx C \exp (-W / k T)$ to give

$$
|\delta \sigma / \sigma|=\left(W / k T^{2}\right)|\delta T| .
$$

For $\mathrm{AgCl}$ the error is $4 \%{ }^{\circ} \mathrm{C}$ at $200{ }^{\circ} \mathrm{C}$ and $2.5 \% /{ }^{\circ} \mathrm{C}$ at the melting point of $455{ }^{\circ} \mathrm{C}$, corresponding to about $0.4 \%$ for a temperature error of $0.1 \% \mathrm{C}$ or slightly larger.

Two conclusions may be drawn at this point. First, the estimated errors in each measured conductivity value may accumulate to about $0.5 \%$. This figure must serve as a general indication of the size of the errors involved, but it is still a bit rough to use in a quantitative chi square test. In order to develop a test to obtain a firmer quantitative estimate, we are greatly assisted by the second conclusion. The per cent error is independent of temperature, to a good first approximation. The greatest variation comes from the factor $1 / T^{2}$ in eq. (24), but even this variation is less than a factor of two over the entire temperature range. Furthermore this apparently systematic trend is doubtlessly overshadowed, at least in part, by the vagueness of the estimates of the other contributions to the total error. Hence we adopt the working hypothesis that all errors $e_{\mathrm{i}}$ amount to some constant per cent, independent of temperature, and proceed in the next section to obtain a quantitative estimate for this constant per cent error

4. Chi square test for distribution of errors. Let us consider first the collection of deviations shown in figures $3 a$ or $3 b$. If we assume that the measurement error of each point results from the accumulated effect of a number of random influences, we expect to have an approximately normal distribution of probabilities for finding an error of any particular size. If we further assume that the fitting function based on our proposed model for the conductivity provides an adequate fit, the deviations shown in the figure should represent solely the effect of the random errors. Casual inspection of figure $3 b$ does show that in this case there is no obvious systematic trend. thereby increasing our confidence in the adequacy of the model, and also indicates a substantially constant per cent error over this more limited temperature range, in agreement with the second conclusion of the preceeding section. In figure $3 a$, on the other hand, there is some reason to question a purely random distribution of deviations.
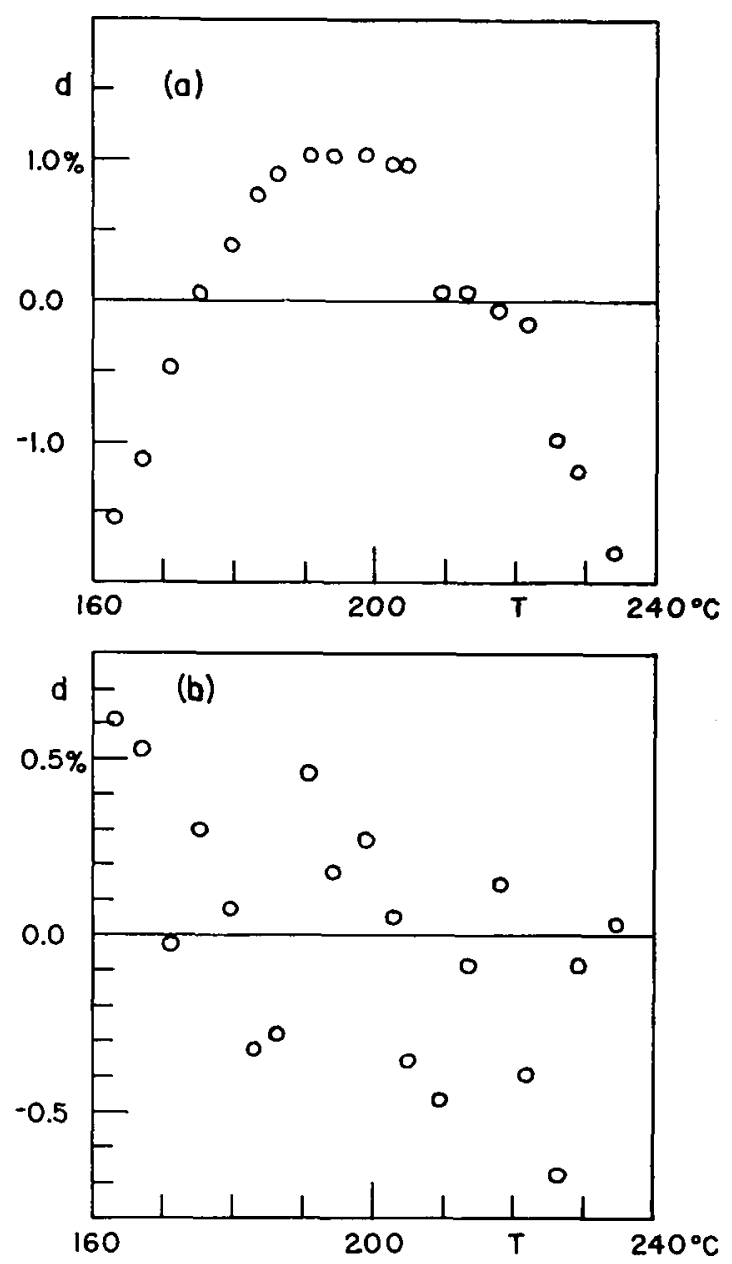

Fig. 3. - Deviation plot for $\mathrm{AgBr}$ (sample $12 \mathrm{ABC}$ ) for the intermediate temperature range where a good fit can be obtained. a) At an early stage of adjusting the parameters in the fitting procedure. $b$ ) At the end of the fitting procedure after the parameters have been adjusted to obtain the best fit. Note the different scale of deviations for part $b$ ).

We are now faced with two questions. 1) How can we decide if a particular set of deviations represents a reasonable sample from a normal error distribution?

2) If it does, what is the width, or standard deviation, of the most suitable normal distribution ? A graphical answer can be presented by constructing a histogram of the actual sample distribution and of the proposed normal distribution, after estimating the appropriate standard deviation from the sample itself (see below). Figure $4 b$ shows rather good agreement of the two distributions, but figure $4 a$ leaves some question about the goodness of fit. The purpose of this section is to develop a chi square test to provide a quantitative assessment of the goodness of fit.

We first state some well-known properties of the 


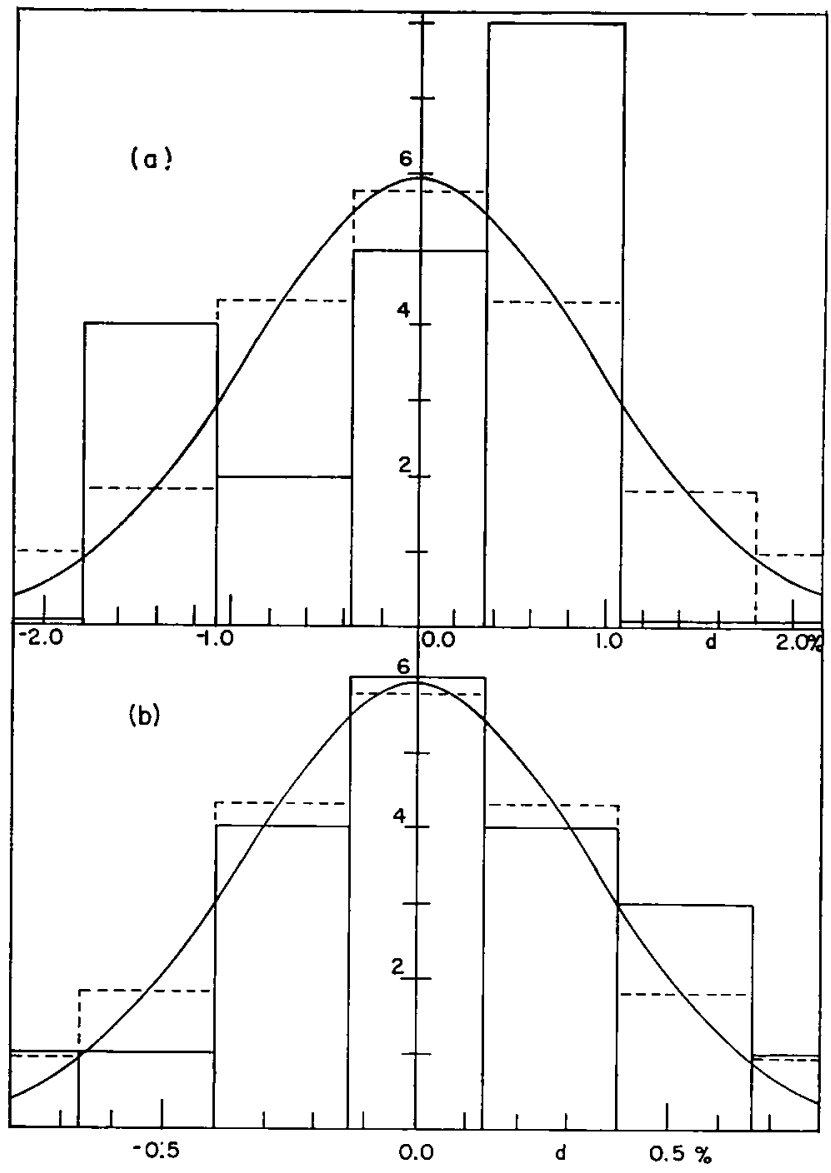

FIG. 4. - Histograms of error distributions. Parts $a$ ) and $b$ ) correspond to the deviations shown in figures $3 a$ (poor fit) and $3 b$ (good fit). The frequencies of occurence obtained from the sample distribution are shown by solid lines. The normal distribution curve is calculated with the maximum likelihood estimate of the standard deviation from eq. (28), and the corresponding frequencies are shown by dotted lines.

normal distribution with mean $m$ and standard deviation $b[21]$.

$$
\begin{aligned}
F(x) & =\left(2 \pi b^{2}\right)^{-1 / 2} \exp \left[-\frac{1}{2}(x-m)^{2} / b^{2}\right] \\
1 & =\int_{-\infty}^{\infty} F(x) \mathrm{d} x, \quad \bar{x}=\int_{-\infty}^{\infty} x F(x) \mathrm{d} x=m \\
x_{r m s}^{2} & =\int_{-\infty}^{\infty}(x-m)^{2} F(x) \mathrm{d} x=b^{2} .
\end{aligned}
$$

A reduced normal distribution with mean 0 and standard deviation 1 may be obtained by substituting

$$
\begin{gathered}
z=(x-m) / b . \\
f(z)=(2 \pi)^{-1 / 2} \exp \left(-\frac{1}{2} z^{2}\right) .
\end{gathered}
$$

We shall also need the integrated distribution function

$$
\Phi(z)=\int_{-\infty}^{z} f(t) \mathrm{d} t,
$$

which has the properties

$$
\begin{aligned}
& \Phi(-\infty)=0.0, \quad \Phi(0)=0.5, \quad \Phi(\infty)=1.0, \\
& \Phi(-z)=1-\Phi(z) .
\end{aligned}
$$

Then the probability that an observation occurs within the range between $x_{1}$ and $x_{2}$ is

$P\left(x_{1}<x<x_{2}\right)=\Phi\left(\frac{x_{2}-m}{b}\right)-\Phi\left(\frac{x_{1}-m}{b}\right)$.

Standard tables of $f(z)$ and $\Phi(z)$ are readily available $[21]$.

The most suitable values of $m$ and $b$ for the proposed normal distribution are obtained from the sample by calculating the maximum likelihood estimates [22], which for a normal distribution are $\left(^{2}\right)$

$$
m=n^{-1} \Sigma_{i} d_{\mathrm{i}}, \quad b^{2}=n^{-1} \Sigma_{i}\left(d_{\mathrm{i}}-m\right)^{2} .
$$

This procedure derives its name from the fact that use of these values for $m$ and $b$ gives the maximum probability of drawing the sample at hand from the proposed distribution. In our examples we find $m$ to be essentially zero because we have already varied the parameters to minimize $\Sigma_{i} d_{i}^{2}$, and therefore we can calculate according to

$$
m=0, \quad b^{2}=n^{-1} \sum_{i} d_{i}^{2} .
$$

Next we select a number of ranges $1,2, \ldots, k, \ldots, g$ of the deviations; in figure $4 b$ the central range is -0.133 to $0.133 \%$, the first range on the right is 0.133 to $0.400 \%$, and the final range is $-\infty$ to $-0.677 \%$ and $0.667 \%$ to $+\infty$. Finally we determine the frequency of occurrence of deviations in each of the ranges. The sample frequencies $n_{k}$ are obtained simply by counting, and the population frequencies $n p_{k}$ are evaluated from eq. (26).

The chi square test compares the sample and population frequencies by means of a quantity [22]

$$
\chi^{2}=\Sigma_{k}\left(n_{k}-n p_{k}\right)^{2} /\left(n p_{k}\right), \quad k=1 \text { to } g \text {. }
$$

Not all of the terms in this sum are independent, however, because first of all the frequencies are normalized, as expressed by either

$$
\Sigma_{k} n_{k}=n \text { or } \Sigma_{k} p_{k}=1 \text {, }
$$

and secondly two parameters of the distribution have been determined from the sample. Hence the number of linearly independent terms in eq. (29), which is the number of degrees of freedom for the chi square test, is

$$
v=g-1-2=g-3 .
$$

If the agreement between the sample and proposed distributions is good, each term in eq. (29) has an expectation value of one, and we expect to find $\chi^{2} \sim v$ in an average case. If the agreement is poor, however, we shall obtain an appreciably larger value for $x^{2}$.

It is not possible to go into a complete description of the theory of the chi square distribution here, but

(2) Note the use of $n$ rather than $n-1$ for determining the standard deviation; this is a particular feature of the maximum likelihood method. 
a few remarks may help to give a general understanding. The quantity $\chi^{2}$ is defined as a sum of squares of independent variables, each variable having a normal probability distribution with $m=0$ and $b=1$ [22]. This condition is satisfied asymptotically in eq. (29) when all $n_{k} \gg 1$, for then the differences between $n_{k}$ and $n p_{k}$ should have a mean of zero and a standard deviation of $\left(n p_{k}\right)^{1 / 2}$, essentially a counting error. Strictly speaking, the distribution of $n p_{k}$ is binomial for small $n_{k}$, but becomes asymptotically Poisson and then normal as $n_{k}$ becomes large. With this definition of $\chi^{2}$, it is not especially difficult to derive the chi square distribution for $v$ degrees of freedom,

$$
k_{v}(x)=\left[2^{1 / 2 v} \Gamma\left(\frac{1}{2} v\right)\right]^{-1} x^{1 / 2 v-1} \mathrm{e}^{-1 / 2 x},
$$

where $\Gamma\left(\frac{1}{2} v\right)$ is the gamma function. It is not particularly important for our purposes to explore further the nature of this distribution, but it is reassuring to know that an explicit expression exists.

For application of the chi square test the most useful information is the probability that $\chi^{2}$ will exceed a specified value $\chi_{\mathrm{p}}^{2}$. If we express this probability by $p$ percent, the relationship between $\gamma_{\mathrm{p}}^{2}$ and $p$ is established by

$$
P\left(\chi^{2}>\chi_{p}^{2}\right)=\int_{\chi^{2} \frac{2}{p}}^{\infty} k_{v}(x) \mathrm{d} x=\frac{p}{100} .
$$

The standard tables [21] list values of $\chi_{\mathrm{p}}^{2}$ for selected entries of $v$ and $p$. If the value of $\chi^{2}$ that we calculate from a sample is greater than $\gamma_{\mathrm{p}}^{2}$, we say that the sample has failed the chi square test at a level of significance of $p$ per cent. The choice of the level of the test is to some extent a matter of judgment, but a reasonable suggestion is to declare that the statistical deviation is almost significant at the $5 \%$ level, significant at the $1 \%$ level, and highly significant at the $0.1 \%$ level [22]. As an illustration, the value of $\%_{p}^{2}$ at the $50 \%$ level is just slightly less than ${ }^{\prime}$, in agreement with the qualitative argument below eq. (30).

We are now in a position to apply the chi square test to the two sets of deviations in figure 4 . In each case the number of degrees of freedom is 3 because there are 6 ranges (remember that the two outermost regions are combined into one range). The calculations proceed according to eq. (29), and the results are shown in table I. For the good fit of figure $4 b$ the result is at greater than the $50 \%$ level. and this is, of course, highly acceptable. For the poor fit of figure $4 a$ the result is at the 1 to $2 \%$ level, which is almost significant according to our previous criterion. The chi square test tells us that even if the deviations in figure $4 a$ were drawn at random from a normal distribution there is approximately a $2 \%$ probability that il value of $\%^{2}$ this large could be obtained exclusively from statistical fluctuations. So if we reject a fit at the 2 " level, we must realize that we run the danger of rejecting a valid sample
TABLE I

Chi square tests for distribution of errors

\begin{tabular}{ccccc} 
& Fig. $4(v=3)$ & \multicolumn{2}{c}{ Fig. $5(v=7)$} \\
$p(\%)$ & $\chi_{\mathrm{p}}^{2} \chi^{2}$ (calc) & \multicolumn{2}{c}{$\chi_{\mathrm{p}}^{2} \chi^{2}$ (calc) } \\
- & \multicolumn{2}{c}{-} & \multicolumn{2}{c}{-} \\
50 & 2.37 & & 6.35 & \\
20 & 4.64 & & 9.80 & \\
& & & & 11.06 \\
10 & 6.25 & & 12.02 & \\
5 & 7.82 & & 14.07 & \\
2 & 9.84 & & 16.62 & \\
& & 9.91 (a) & & \\
1 & 11.34 & & 18.48 & \\
0.1 & 16.27 & & 24.32
\end{tabular}

in $2 \%$ of the cases. But we may also obtain a large value of $\chi^{2}$ if the deviations are not genuinely random, and figure $3 a$ confirms our suspicions in this case.

It is desirable for strict reliability of a chi square test to have each $n_{k}$ at least as large as 5 , and 10 would be better. In figure 4 , however, the frequencies range from 0 to 8 because there are only 19 points in the sample. For this reason another case based on 37 points is shown in figure 5 and table $\mathrm{I}$. Although the agree-

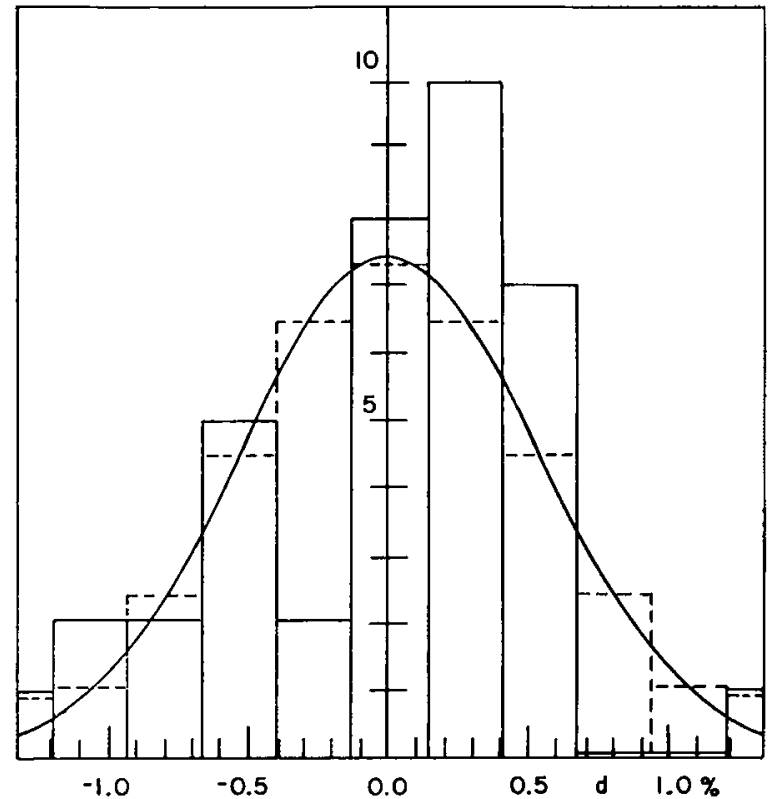

FlG. 5. - Histogram of error distribution for $\mathrm{AgCl}$ (sample $A B$ 12-2) for the intermediate temperature range. See figure 4 for interpretation of the figure.

ment in figure 5 does not appeatr to be as favorable as that in figure $4 b$, the value of $\gamma^{2}$ in table I corresponds to the 10 to $20 \%$ level, which is usually considered acceptable. This example begins to show some of the real power of the chi syuare test; especially for larger numbers the statistical test offers a more 
quantitative and precise basis for judging the goodness of fit than can be obtained with the human eye.

There are several important conclusions from the results of this section. In the first place we have shown that the deviations in cases of good fits, figures $4 b$ and 5 , are essentially random and have a normal distribution as expected. In addition, the width $b$ of this distribution gives us a quantitative estimate of the standard deviation. In the silver halide work [14], for instance, chi square values at the $50 \%$ level are found for sets of deviations using pooled data from several different samples of each kind of crystal. Furthermore, the values of $b=0.39 \%$ for $200^{\circ}$ to $300{ }^{\circ} \mathrm{C}$ in $\mathrm{AgCl}$ and $0.43 \%$ for $160^{\circ}$ to $240{ }^{\circ} \mathrm{C}$ in $\mathrm{AgBr}$ are in excellent conformity with the experimental estimates of section 3. Hence these results can serve as a quantitative basis for considering extension of the conductivity fit to a wider temperature range.

5. Chi square test for conductivity fit. - The procedure for making a conductivity fit is expressed schematically by eq. (17), and the quality of the fit that is obtained is displayed graphically by deviation plots such as those in figure 3 . In the general case when the errors $e_{\mathrm{i}}$ are known to be different for different points, the terms in the sum of the squares of the deviations should be weighted by $e_{i}^{-2}$ in order to obtain the maximum likelihood that the fitting function will represent the observed data [23]. Therefore as a generalization of eq. (19) we define

$$
\chi^{2}=\Sigma_{i} d_{\mathrm{i}}^{2} / e_{\mathrm{i}}^{2}, \quad i=1 \text { to } n .
$$

The best fit is then found by varying the $r$ parameters in the fitting function to minimize $\chi^{2}$. If the deviations $d_{i}$ do, in fact, follow a normal distribution with mean 0 and standard deviation $e_{i}$, we see that the sum defined by eq. (33) conforms to the basic requirements for a chi square distribution described in the last section. Since not all of the terms are linearly independent, however (if we had $r=n$ we could obtain an exact fit at all points and $\chi^{2}$ would be identically zero !), the number of degrees of freedom is only $v=n-r$ as given by eq. (21).

A quantity closely related to $\chi^{2}$ is the variance of the fit. In accordance with the weighted sum of eq. (33) we introduce raw weights $W_{\mathrm{i}}=e_{\mathrm{i}}^{-2}$ and normalized weights $w_{\mathrm{i}}$, so that $\Sigma_{i} w_{\mathrm{i}}=n$, by defining

$$
w_{\mathrm{i}}=n W_{\mathrm{i}} /\left(\Sigma_{i} W_{\mathrm{i}}\right)=e_{0}^{2} / e_{\mathrm{i}}^{2} .
$$

The final convenient form for the reduced weights is obtained by defining an average weight

$$
\frac{1}{e_{0}^{2}}=W=\frac{1}{n} \Sigma_{i} W_{\mathrm{i}}=\frac{1}{n} \Sigma_{i} \frac{1}{e_{\mathrm{i}}^{2}}
$$

A generalization of eq. (20) for the variance of the fit is

$$
s^{2}=(n-r)^{-1} \sum_{i} w_{\mathrm{i}} d_{\mathrm{i}}^{2},
$$

and by comparison to eq. (33) we find

$$
s^{2}=(n-r)^{-1} e_{0}^{2} \chi^{2} .
$$

The size of $s^{2}$ gives us an overall indication of the largeness of the deviations from the fit. We can also estimate the overall root-mean-square error $E$ that should be caused by the experimental errors of the individual points by calculating a suitable weighted average.

$$
E^{2}=n^{-1} \Sigma_{i} w_{i} e_{\mathrm{i}}^{2}=e_{0}^{2} .
$$

The result that $E=e_{0}$, which is readily obtained from eq. (34) and (35), is not surprising. The ratio of $s^{2}$ to $e_{0}^{2}$ now gives a good measure of the average size of the deviations from the fit compared to the average size of the experimental errors [23]. We find

$$
s^{2} / e_{0}^{2}=(n-r)^{-1} \chi^{2}=\chi_{v}^{2},
$$

where the reduced chi square is defined simply by [24]

$$
\chi_{v}^{2}=\chi^{2} / v
$$

The variance of the data $e_{0}^{2}$ is characteristic of the spread of the data from the experimental errors and is not descriptive of the fit, but the variance of the fit $s^{2}$ is characteristic of both the spread of the data and the accuracy of the fit. Thus if a good fit is obtained, $s^{2}$ should agree well with $e_{0}^{2}$, and we expect to find $\%^{2} \sim 1$, corresponding to the $50 \%$ level. If the fitting function is not appropriate for describing the data, then the deviations will be larger, and we shall find appreciably larger values for $\chi_{v}^{2}$. The statistical significance of a large value of $\gamma^{2}$ is obtained by comparing it to $\gamma_{v}^{2}(p)$ for a desired level of significance $p$ per cent, as described in the preceding section.

There are two different circumstances for applying a chi square test to the problem of fitting a function to a set of measured points. In the first case the errors $e_{i}$ are known, but not necessarily equal, for each of the experimental points. A typical example would be a collection of diffusion coefficients measured at various temperatures. An independent assessment can then be made of the error in each point arising from such sources as the slope of the diffusion profile, measurement of sample dimensions, temperature measurements, and corrections for heating and cooling times [20]. Another example is furnished by an angular correlation experiment in nuclear physics, where the angular cross section for some nuclear reaction is determined by measuring the counting rate for various angular positions of the detector. In this case the parameters in the fitting function are items like the spin and parity of an excited nuclear state under investigation, and a major factor in the errors is simply the statistical counting error. It was, in fact, the experience of sitting through many years of colloquium talks in which nuclear physicists repea- 
tedly mentioned the commonplace use of chi square tests for such problems that convinced the author of the desirability of adapting these tests to ionic conductivity problems. In these circumstances the quantities $e_{0}^{2}$ and $s^{2}$ can be calculated directly from the data according to eq. (35) and (36), or $\chi^{2}$ can be evaluated immediately from eq. (33), and the application of the test is straightforward.

The second case involves a situation, such as we encounter with a conductivity fit, when an independent estimate of the error for each data point is not available. If we have some assurance that the errors are constant, i. e., $e_{i}=e_{0}$, as we have demonstrated in section 3 for the conductivity measurements, we find that all normalized weights $w_{i}=1$ and can calculate $s^{2}$ from eq. (20). In order to calculate $\chi^{2}$ from the simplification of eq. (33), however,

$$
\chi^{2}=e_{0}^{-2} \Sigma_{i} d_{i}^{2}
$$

or to obtain $\chi_{y}^{2}$ from eq. (39), we need to have an independent estimate of $e_{0}$. Even if the value of $e_{0}$ is not known, we can still minimize $s^{2}$ to obtain the best fit, and we can certainly suspect that something is wrong with the model when $s^{2}$ becomes noticeably larger as the temperature range is extended. In order to determine the statistical significance of such a change, however, we must have values of $\chi^{2}$ or $\%^{2}$, and that is one of the important reasons for testing the deviations in section 4.

We can apply the chi square test to conductivity fits for the examples in figures 4 and 5 by using the values of $e_{0}=b$ determined in section 4 . The fitting function of eq. (14) is used for these fits for the pure crystal. Since $k$ and $\rho_{2}$ are constrained to the variation obtained from diffusion and doped conductivity experiments, respectively [14], and since $h_{\mathrm{F}}$ and $s_{\mathrm{F}}$ are held fixed at the average values previously obtained from impure crystal fits, inspection of eq. (14) shows that only two parameters, namely, $\Delta / l_{s}$ and $\Delta s_{v}$, remain free to vary. A good deal of smoothing of the data has already been accomplished, however, in the impure crystal fits, and we have therefore, somewhat arbitrarily, taken the number of degrees of freedom as $r=n-6$. Since $v=31$ for the case of figure 5, and since many chi square tables have a maximum entry of $v=30$, it may be necessary to use asymptotic formulas to calculate $x^{2}(p)$. Two expressions are [22]

$\chi_{v}^{2}(p)=\left[1-(2 / 9 v)+x_{p}(2 / 9 v)^{1 / 2}\right]^{3}, \quad v>30$,

$\chi_{v}^{2}(p)=(2 v)^{-1}\left[(2 v-1)^{1 / 2}+x_{p}\right]^{2}, v>100$,

where $x_{p}$ is obtained from the normal distribution by the condition

$$
P\left(x>x_{p}\right)=1-\Phi\left(x_{p}\right)=p / 100
$$

The results are shown in table II in terms of the reduced chi square, which is convenient for this
TABLE II

Chi square tests for conductivity fits. The reduced chi square $\chi_{v}^{2}=\chi^{2} / v$ is shown.

\begin{tabular}{ccclc} 
& \multicolumn{2}{l}{ Fig. $4(v=13)$} & \multicolumn{2}{l}{ Fig. $5(v=31)$} \\
$p(\%)$ & $\chi_{v}^{2}(p)$ & $\chi_{v}^{2}($ calc $)$ & $\chi_{v}^{2}(p)$ & $\chi_{v}^{2}(\mathrm{calc})$ \\
50 & - & - & - & - \\
20 & 0.95 & $0.95(\mathrm{~b})$ & 0.98 & \\
10 & 1.31 & & 1.21 & \\
5 & 1.52 & & 1.34 & \\
2 & 1.72 & & 1.46 & \\
1 & 1.96 & & 1.60 & \\
0.1 & 2.13 & & 1.70 & \\
& 2.66 & & 1.99 &
\end{tabular}

section. For the good fit of figure $3 b$ the calculated value of $\%^{2}$ is at the $50 \%$ level, indicating a highly satisfactory fit. For the poor fit of figure $3 a$, in contrast, the calculated value of $\psi^{2}$ is far beyond the $0.1 \%$ level, a completely untenable result that confirms our worst fears about the systematic trends indicated by the deviation plot. For the case of figure 5 the calculated value of $\chi^{2}$ is slightly beyond the $0.1 \%$ level, suggesting that some failure of the model is beginning to appear. The results in table III, however,

\section{TABLE III}

Effect of extending temperature range on the conduc-

\begin{tabular}{|c|c|c|c|c|c|}
\hline $\begin{array}{c}T_{2} \\
\left({ }^{\circ} \mathrm{C}\right)\end{array}$ & $n$ & $x^{2}$ & $\begin{array}{l}p \\
(\%)\end{array}$ & $\begin{array}{l}\Delta h_{\mathrm{v}} \\
(\mathrm{eV})\end{array}$ & $\begin{array}{c}\Delta s_{\mathrm{V}} \\
(\mathrm{eV} / \mathrm{K})\end{array}$ \\
\hline - & - & - & - & -- & - \\
\hline 291.41 & 34 & 1.07 & to 50 & 0.275 & $-0.5523 \times 10^{-4}$ \\
\hline 293.11 & 35 & 1.47 & to 5 & 0.275 & $-0.5519 \times 10^{-4}$ \\
\hline 296.03 & 36 & 1.97 & 0.1 to 1 & 0.275 & $-0.5517 \times 10^{-4}$ \\
\hline 299.23 & 37 & 2.38 & $<0.1$ & 0.274 & $-0.5514 \times 10^{-4}$ \\
\hline
\end{tabular}
tivity fit for $\mathrm{AgCl}$ (sample $\mathrm{AB} \mathrm{12-2)} \mathrm{from} \mathrm{ref.} \mathrm{[25].}$

show that a greatly improved value of $\chi^{2}$ can be obtained by removing only two or three points at the high temperature end of the range.

The most important use of the chi square test for the silver halide work is to establish the upper (and lower) temperature limits of the intermediate temperature range where a good fit can be obtained. After an initial suitable range is established by the procedures of section 4, additional data points are added one by one at the upper end of the temperature range in steps of about 5 "C. For each new point a new set of parameters is obtained to give the best fit, and a new value of $x^{2}$ is calculated. Typical results are shown in figure 6 and table III [25]. There is a striking increase in $\%^{2}$ as the temperature range begins to encroach upon the high temperature region where the conductivity anomaly appears. A data point is usually rejected when the calculated $\%_{*}^{2}$ exceeds the $1 \%$ level. The rise in $\%_{w}^{2}$ is so sudden that the ambiguity 


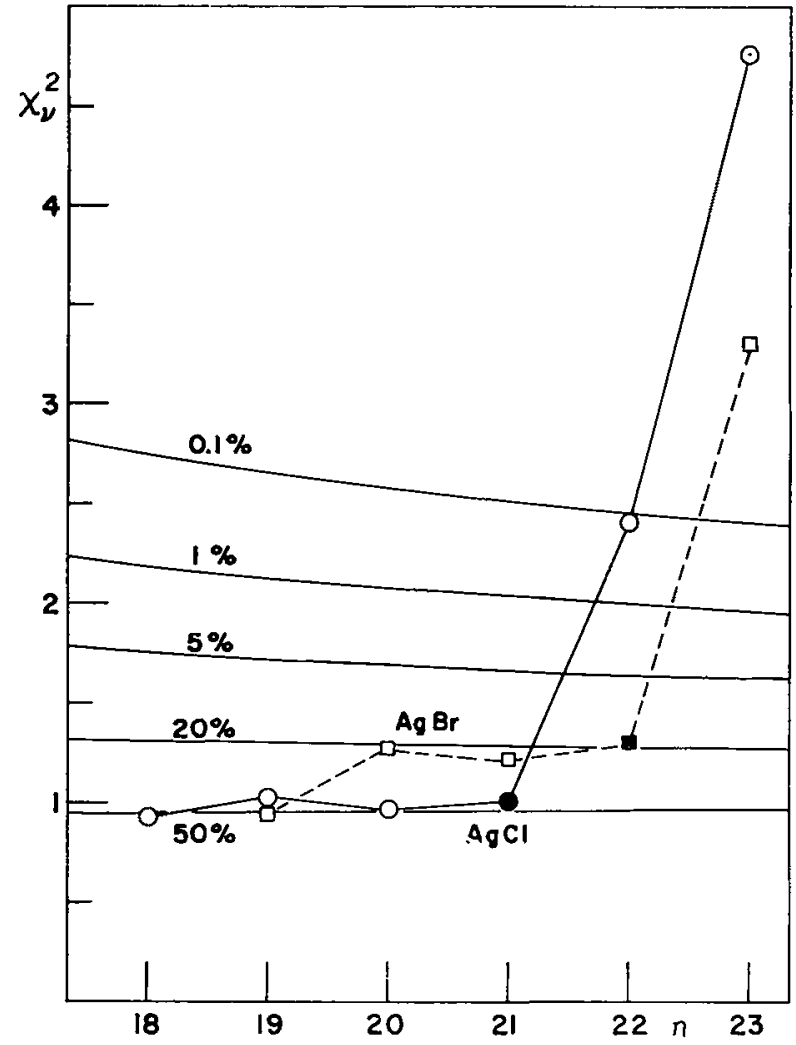

Flg. 6. - Chi square test for conductivity fit for $\mathrm{AgCl}$ (sample GR 12-2) and for $\mathrm{AgBr}$ (sample $12 \mathrm{ABC}$ ) [14]. As additional data points are included at the high temperature end of the range, the reduced chi square $z_{v}^{2}$ increases markedly. The solid points indicate the upper temperature limit selected for determination of the thermodynamic parameters. The solid lines show levels of significance for $n-6$ degrees of freedom, where $n$ is the number of data points.

in selecting the upper temperature limit is at most one data point or $5^{\circ} \mathrm{C}$. Furthermore the last two columns of table III show that there is no significant influence on the parameter values, even if this limit is exceeded by one or two points. A similar procedure is also employed at the lower end of the temperature range, where sometimes one or two points are rejected for the impure crystal fits.

6. Conclusion. - The final results for $\mathrm{AgCl}$ are shown in figure 7 ; a similar plot for $\mathrm{AgBr}$ shows an even larger anomaly [14]. The conductivity anomaly is expressed in terms of an extra interaction energy $\Delta g_{\text {ex }}$ such that

$$
\sigma_{\text {expt }}=\sigma_{\text {theory }} \exp \left(\Delta g_{\text {ex }} / 2 k T\right) .
$$

Since the Debye-Hückel-Lidiard corrections are already included in calculating $\sigma_{\text {thcory }}$, the total excess free energy is

$$
\Delta g=\Delta g_{\mathrm{DII}}+\Delta g_{\mathrm{cx}} .
$$

The assumption in this procedure is that all thermodynamic parameters $\left(h_{\mathrm{F}}, s_{\mathrm{F}}, \Delta h_{v}, \Delta s_{\mathrm{v}}\right.$, etc.) remain independent of temperature in the high temperature

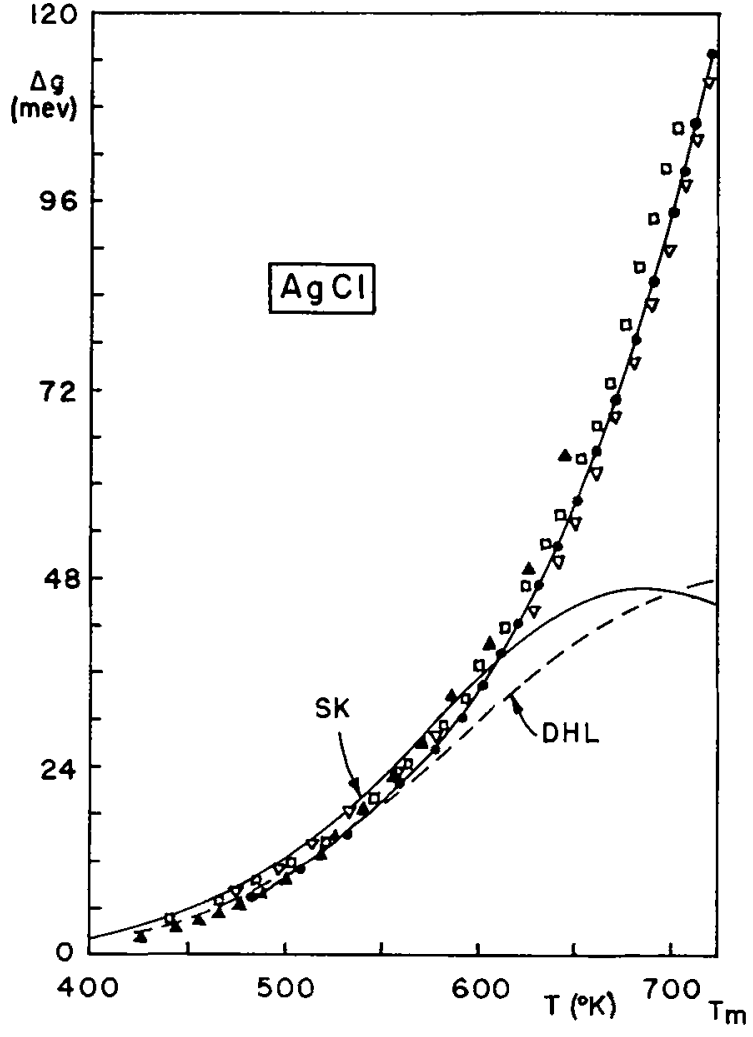

Fig. 7. - Anomalous free energy for $\mathrm{AgCl}$. $, \boldsymbol{\Delta}, \square, \nabla$. experimental points [14]. DHL, calculated from the DebyeHückel-Lidiard theory [1]. SK, improved calculation of Sevenich and $\mathrm{K}$ liewer [26].

region, and that all of the enhanced conductivity is due to an enhancement of the defect concentrations because of $\Delta g_{\mathrm{c} x}$. It is clear, therefore, that it is vital to establish reliable values for the parameters in the intermediate temperature region before proceeding with the extrapolation to high temperatures. The figure shows that for at least $100^{\circ} \mathrm{C}$ below the melting point neither the first order DHL theory [I] nor the higher order calculation of Sevenich and Kliewer [26] is adequate. There must be some other physical process coming into play, and the next plausible suggestion is a general softening of the lattice, which would lower $\Delta g_{v}, \Delta g_{1}, \Delta g_{2}$ along with $g_{F}$ and would thereby enhance both the mobilities and the concentrations [2], [14]. [19], [27].

There are two final remarks about the use of the chi square test. The first is that it does not reveal any startlingly new features that are not apparent in a qualitative sense on the basis of some experience, judgment, and common sense. The high temperature anomaly is obvious from the simple deviation plot of figure 2, and the appearance of systematic deviations is often apparent even in the less dramatic deviation plots like figure $3 a$. The outline of this work was well established on just such grounds, in fact, long before we knew how to use a chi square test. But there is sometimes an uneasy feeling in saying, "That fit looks good, and that other fit looks bad. " The real merit of the chi square test is that it 
allows a quantitative statement with statistical meaning to be made about the goodness of fit. "This fit is so bad that it would occur only $1 \%$ of the time because of purely statistical fluctuations. ")

The other point is that the interpretation of a chi square test must not be taken too literally; a certain amount of judgment is still required. The determination of $e_{0}$, for instance, is subject to some subjective influence both from the selection of data and from determination of the basic temperature range for the initial good fit. When dealing with random errors, we must also accept the inevitable occurrence of fluctuations, so that any mathematically rigorous statement of the outcome of a chi square test is necessarily probabilistic in nature. And even the mathematical formulation of the chi square test for fitting a distribution is only approximately correct when fairly small numbers of observations are involved in each interval. These are reasons why a rejection is seldom made at the 5 or $10 \%$ level; it is safer to allow for some margin of error [22]. Hence the precise level of significance (e. g., $p=7.13 \%$ !) is not of much import, but it is meaningful when the level changes from better than $20 \%$ to less than $0.1 \%$.

We see, then, that the chi square test has been of considerable usefulness in the work on the silver halides. It has provided a reliable quantitative estimate of the average experimental error, and it has given a rather sharply defined limit to the temperature range in which the model is adequate. In this latter form the chi square test should be useful for attacking many of the questions mentioned in the introduction. Whenever the chi square test shows that the model is incapable of providing a good fit, some new effect must be sought out. It may be the appearance of a new defect mechanism, such as vacancy triplets in $\mathrm{KCl}$; it may be the occurrence of a new type of phenomenon, such as softening of the lattice in $\mathrm{AgBr}$; it may even be a more mundane matter involving a hitherto unsuspected systematic error, such as a faulty thermocouple calibration. Whatever the source may be, the chi square test can point the finger and leave the elucidation to the human ingenuity of the investigator.

\section{References}

[1] Lidiard, A. B., Encyclopedia of Physics S. Flügge, ed., Vol. XX, p. 246 (Springer-Verlag, Berlin) 1957.

[2] Teltow, J., Annln. der Phys. 5 (1950) 63;Z. Phys. Chem. 195 (1950) 213.

[3] Dreyrus, R. W. and Nowick, A. S., J. Appl. Phys. 33 (1962) 473; Phys. Rev. 126 (1962) 1367.

[4] Kirk, D. L. and Pratt, P. L., Proc. Br. Ceram. Soc. 9 (1967) 215.

[5] Beaumont, J. H. and Jacobs, P. W. M., J. Chem. Phys. 45 (1966) 1496

[6] Dawson, D. K. and Barr, L. W., Phys. Rev. Lett. 19 (1967) 844

[7] Allnatt, A. R. and Pantelis, P., Solid State Commum. 6 (1968) 309.

[8] Allnatt, A. R., Pantelis, P., Sime, S. J., J. Phys. C 4 (1971) 1778

[9] Chandra, S. and Rolfe, J., Can. J. Phys. 48 (1970) 412.

[10] Chandra, S. and Rolfe, J., Can. J. Phys. 49 (1971) 2098.

[11] Chandra, S. and Rolfe, J., Can. J. Phys. 48 (1970) 397.

[12] Chandra, S. and Rolfe, J., Can. J. Phy's. 51 (1973) 236.

[13] Corısh, J. and Jacors, P. W. M., J. Phys. \& Chem. Solids 33 (1972) 1799.

[14] Abongye, J. and Friauf, R. J., Phy's. Rev., to be published.

[15] Fuller, R. G. and Reilly, M. H., Phys. Rev. Lett. 19 (1967) 113.
[16] Nelson, V. C. and Friauf, R. J., J, Phys. \& Chem. Solids 31 (1970) 825.

[17] Fuller, R. G., Marquardt, C. L., Reilly, M. H., Wells, J. C., Phys. Rev. 176 (1968) 1036.

[18] Fuller, R. G. and Rellly, M. H., J. Phys. \& Chem. Solids 30 (1969) 457.

[19] Erert, I. and Teltow, J., Annhn. der Phys. 15 (1955) 268.

[20] Friauf, R. J., American Institute of Physics Handbook D. E. Gray, ed., 3rd ed., p. 9-74 (McGraw-Hill, New York) 1972.

[21] Burington, R. S. and MAY, D. C., Handbook of Probability and Statistics (Handbook Publ., Sandusky, Ohio) 1953

[22] Cramer, H., Elements of Probability Theory (Wiley, New York) 1955.

[23] Bevington, P. R., Data Reduction and Error Analysis for the Physical Sciences (McGraw-Hill, New York) 1969.

[24] BEYER, W. H., editor, Handbook of Tables for Probability and Statistics (Chemical Rubber Co., Cleveland, Ohio) 1966.

[25] Aboagye, J. K., Thesis, University of Kansas (1972).

[26] Sevenich, R. A. and Kliewer, K. L., J. Chem. Phys. 48 (1968) 3045.

[27] Müller, P., Phys. Stat. Solid. 12 (1965) 775.

\section{DISCUSSION}

A. B. LIDIARD. - I believe the sort of analysis made by Friauf and Aboagye is very important. As the authors themselves indicate, the discrepancy arising at high temperatures indicates that a physical effect is left out of the equations used for the analysis. I believe it is very likely that the assumption that the formation enthalpy of Frentiel defects, $h_{F}$, is independent of temperature is too restrictive. Thus in the
Ag-halides the static dielectric constant, $\varepsilon_{0}$, varies rapidly with temperature at high temperatures and from such variations will very likely follow significant changes in the formation free energy comparable, to the $0.1 \mathrm{eV}$ indicated by Friaur's analysis. This is shown not only by the classical continuum formula of Jost which indicates that $g_{\mathrm{F}}$ is proportional to $\left(1-1 / \varepsilon_{0}\right)$ but also by recent detailed calculations using the 
quasi-harmonic model for the Caesium halides (Müller and Norgett, in press).

L. SLIFKIN. - Are the conductivity data of $\mathrm{AgCl}$ well fitted by involving cooperative processes.

P. W. M. JACOBS. - Yes, we did obtain excellent fits to the conductivity of $\mathrm{AgCl}$ over the whole temperature range (J. Corish and P. W. M. Jacobs, J. Phys. \& Chem. Solids 31 (1972) 1799. This analysis illustrates one of the features of computer fitting, that the parameters for the minor contribution to the conductivity (in this case the non-collinear interstitialcy motion) are determined with low accuracy if the model is not perfectly correct. The relatively poor agreement between the NCL parameters for crystals of different purity ( 5 and $31 \mathrm{ppm}$ ) emphasizes that something peculiar is happening at high temperatures in silver chloride. 